\title{
Patients with no preoperative back pain have the best outcome after lumbar disc herniation surgery
}

\author{
Freyr Gauti Sigmundsson ${ }^{1} \oplus \cdot$ Anders Joelson $^{1} \cdot$ Fredrik Strömqvist $^{2}$
}

Received: 13 June 2021 / Revised: 9 September 2021 / Accepted: 11 October 2021 / Published online: 27 October 2021

(c) The Author(s) 2021

\begin{abstract}
Purpose Most patients with lumbar disc herniations requiring surgery have concomitant back pain. The purpose of the current study was to evaluate the outcome of surgery for lumbar disc herniations in patients with no preoperative back pain (NBP) compared to those reporting low back pain (LBP).

Methods 15,418 patients surgically treated due to LDH with primary discectomy from 1998 until 2020 were included in the study. Self-reported low back pain assessed with a numerical rating scale (NRS) was used to dichotomize the patients in two groups, patients without preoperative back pain (NBP, NRS $=0, n=1333,9 \%$ ) and patients with preoperative low back pain (LBP, NRS $>0, n=14,085,91 \%$ ). Patient reported outcome measures (PROMs) collected preoperatively and one-year postoperatively were used to evaluate differences in outcomes between the groups.

Results At the one-year follow-up, $89 \%$ of the patients in the NBP group were completely pain free or much better compared with $76 \%$ in the LBP group. Significant improvement regarding leg pain was seen in all measured PROMs in both groups oneyear after surgery. In the NBP group, 13\% reported clinically significant back pain (NRS difference greater than Minimally Clinical Important Difference (MICD)) at the one-year follow-up.

Conclusions Patients without preoperative back pain are good candidates for LDH surgery. 13\% of patients without preoperative back pain develop clinically significant back pain one-year after surgery.
\end{abstract}

Keywords Lumbar disc herniation $\cdot$ Back pain $\cdot$ Leg pain $\cdot$ Outcome $\cdot$ Discectomy

\section{Introduction}

Lumbar disc herniation (LDH) is one of the most common indications for spine surgery [1]. Several studies have evaluated different aspects of LDH, both the natural course and the surgical- and non-surgical treatment options [2-4]. LDH has generally a favorable outcome with non-operative treatment, but $5-10 \%$ of all patients may require surgery to relieve symptoms. The main indication for LDH surgery is to alleviate sciatica and most often patients are offered LDH surgery when sufficient relief of leg pain is not experienced

Freyr Gauti Sigmundsson freyr.gauti-sigmundsson@oru.se

1 Department of Orthopedics, Orebro University School of Medical Sciences and Orebro University Hospital, Orebro, Sweden

2 Clinical and Molecular Osteoporosis Research Unit, Departments of Clinical Sciences and Orthopedics, Lund University, Skane University Hospital, Malmö, Sweden after 8-12 weeks of non-surgical treatment. Most patients with LDH requiring surgery have concomitant back pain [1], which can preexist the sciatica or be present at the same time. Several studies have evaluated back pain in different context of the LDH surgical- and non-surgical setting, mainly its preoperative mean magnitude, change following surgical treatment and effect on quality of life [5-10].

To our knowledge, no study has yet focused on the subcategory of operated LDH patients where preoperative back pain is totally absent. The aim of this study was, by using prospectively collected data from a nationwide spine surgical register, to evaluate the outcome of LDH surgery in patients with no preoperative back pain, to describe their preoperative condition and the surgical outcome as compared to the LDH population with coexisting back pain. This knowledge may be valuable for clinicians, to facilitate better information to patients scheduled for LDH surgery. Achieving realistic preoperative expectations with regards to the surgical outcome leads to higher degree of patient satisfaction after surgery $[11,12]$. 


\section{Material and methods}

All patients surgically treated due to LDH with primary discectomy (with or without microscopic assistance) from 1998 until 2020 were identified in Swespine, the National Quality Registry for Spine Surgery. Swespine is a patient-based register and has previously been thoroughly described and validated [1]. The Swespine is a register with no exclusion criteria that welcomes all departments performing spine surgery in Sweden to register preoperative, perioperative and one-, two-, five and ten-years postoperative data. The register covers $90 \%$ of spine operations performed in Sweden. In the standard Swespine protocol, the patients report their age, gender, smoking habits, preoperative duration of backand leg pain, consumption of analgesics, walking distances, the validated patient reported outcome measures (PROMs) Numerical Rating Scale (NRS) for back- and leg pain, quality of life by the EuroQol 5 dimensions (EQ-5D) (UK value set) and disability by the Oswestry Disability Index (ODI). At the one-year postoperative follow-up, the patients also report subjective satisfaction rate on a Likert scale (Dissatisfied, Undecided and Satisfied). The Swespine protocol also has a transition question for evaluation of leg pain outcome: "How is your leg pain/sciatica today as compared to before the surgery?". The response options are (0) No leg pain before the surgery, (1) Completely pain free, (2) Much better, (3) Somewhat better, (4) Unchanged, and (5) Worse [13].

All patients underwent open discectomy with or without surgical microscope by surgeons with varying experience, and in departments ranging from rural to university as well as private spine units. Inclusion in the register is voluntarily, and when patients are registered, they accept that data are used for scientific purposes and presented in publications. Approval for the study was given by the regional ethical review board (Dnr 2017/158).

We used IBM SPSS statistics ${ }^{\circledR}$ version 26 in the statistical calculations and data are presented as numbers $(n)$, means \pm standard deviations (SD) or proportions (\%) and as pain level according to NRS, quality of life by EQ-5D and ODI as means with $95 \%$ confidence intervals (CI) within brackets. Group comparisons were performed using Student's t-test or paired t-tests for means and $\mathrm{chi}^{2}$-test for group comparisons in ordinal data. The level of statistically significant difference was set to $p<0.05$.

\section{Results}

21,729 patients operated for LDH were identified in Swespine. 6311 patients did not have both pre- and one-year postoperative back pain reported and were hence excluded.
1333 patients (9\%) with no preoperative back pain were identified (NRS $=0)$, and all other patients $(\mathrm{NRS}>0$, $n=14,085$ ) operated for LDH during the same period were used for group comparisons.

Baseline data regarding age, gender distribution, smoking status or preoperative duration of leg pain are presented in Table 1. Patients with NBP had preoperatively a better selfestimated walking distance, a lower consumption of analgesics, less leg pain (Table 1), better quality of life (EQ-5D) as well as less disability by ODI (Table 2 , all statistically significant). At one-year postoperative follow-up, the NBP group consumed less analgesics, had a better self-estimated walking distance, lower back- and leg pain (NRS) as well as better quality of life (EQ-5D) and less disability in terms of the ODI (Tables 1 and 2). Further analysis of the demographics of patients who were preoperatively back pain free but at the one-year follow-up presented with clinically significant back pain is presented in Table 3. The results showed female sex and smoking were associated with increased risk for having clinically significant back pain at the one-year follow-up. Patients that developed clinically significant back pain at the one-year follow-up had preoperatively more disability in terms of ODI and lower quality of life in terms of the EQ-5D than patients that did not develop clinically significant back pain (Table 3).

Significant improvement was seen in all measured PROMs in both groups oneyear after surgery (Table 4). Back pain improved significantly in the LBP group oneyear after surgery; however, in the NBP group, back pain was minimally increased at the one-year follow-up (preoperative NRS 0 vs postoperative NRS $=1, p<0.001$ ), Only $13 \%$ of the patients that experienced increased back pain had back pain > MCID (NRS > 2.4) [14] at one-year postoperative follow-up. In the LBP group the corresponding portion that experienced increased back pain (back pain $>$ MCID) (NRS > 2.4) was 39\%. $73 \%$ of the patients in the NBP group achieved an improvement in terms of leg pain exceeding MCID (MCID > 3.4 [14]) for leg pain in LDH surgery, and the corresponding figure in the LBP group was $71 \%(p<0.001)$. In the NBP group, $87 \%$ of the patients were completely pain free or much better (leg pain transition question response options 1 and 2 ), and the corresponding number in the LBP group was $76 \%(p<0.001)$. In the NBP group, $87 \%$ of the patients were satisfied with the surgical outcome at one-year postoperative, and the corresponding figure in the LBP group was $77 \%(p<0.001)$. 
Table 1 Preoperative and one-year postoperative data regarding age, smoking, duration of back- and leg pain, consumption of analgesics and walking distances

\begin{tabular}{|c|c|c|c|c|c|c|}
\hline & \multicolumn{3}{|c|}{ Preoperatively } & \multicolumn{3}{|c|}{ One-year follow-up } \\
\hline & NBP & LBP & $p$ value & NBP & LBP & $p$ value \\
\hline Age & $45 \pm 13$ & $45 \pm 13$ & 0.031 & NA & NA & NA \\
\hline Gender (M/F) & $59 / 41$ & $55 / 45$ & 0.001 & NA & NA & NA \\
\hline Smoking (\%) & 13 & 16 & 0.051 & NA & NA & NA \\
\hline \multicolumn{7}{|c|}{ Duration of leg pain (\%) } \\
\hline Had no leg pain & 1 & 1 & & & & \\
\hline$<3$ months & 20 & 16 & & NA & NA & NA \\
\hline$>3-12$ months & 59 & 56 & $<0.001$ & & & \\
\hline$>12-24$ months & 12 & 15 & & & & \\
\hline$>24$ months & 8 & 12 & & & & \\
\hline \multicolumn{7}{|c|}{ Analgesics consumption (\%) } \\
\hline None & 17 & 11 & & 74 & 53 & \\
\hline Intermittent & 58 & 28 & $<0.001$ & 19 & 31 & $<0.001$ \\
\hline Regular & 24 & 60 & & 6 & 15 & \\
\hline \multicolumn{7}{|l|}{ Walking distance (\%) } \\
\hline$<100 \mathrm{~m}$ & 30 & 30 & & 1 & 3 & \\
\hline$>100$ to $500 \mathrm{~m}$ & 18 & 20 & 0.011 & 3 & 7 & $<0.001$ \\
\hline$>500$ to $1000 \mathrm{~m}$ & 14 & 15 & & 5 & 10 & \\
\hline$>1000 \mathrm{~m}$ & 37 & 32 & & 90 & 79 & \\
\hline
\end{tabular}

Data are presented as mean (SD) or proportions (\%). NA = not applicable. NBP (No back pain) refers to patients without any preoperative back pain according to NRS $(n=1.333)$ and LBP (Low back pain) refers to patients with preoperative back pain NRS $>0(n=14,085)$

Table 2 Preoperative and one-year postoperative data regarding NRS as estimation of back and leg pain, EQ-5D and SF-36 as estimation of quality of life and ODI as estimation of disability

\begin{tabular}{|c|c|c|c|c|c|c|}
\hline & \multicolumn{3}{|l|}{ Preoperatively } & \multicolumn{3}{|c|}{ One-year follow-up } \\
\hline & $\mathrm{NBP}$ & LBP & $p$ value & NBP & LBP & $p$ value \\
\hline \multicolumn{7}{|l|}{ NRS } \\
\hline Back pain & $0(0,0)$ & $5.1(5.1,5.2)$ & $<0.001$ & $1.0(0.9,1.0)$ & $2.5(2.5,2.6)$ & $<0.001$ \\
\hline Leg pain & $6.1(6.0,6.3)$ & $6.2(6.8,6.9)$ & $<0.001$ & $1.1(1.0,1.2)$ & $2.1(2.1,2.2)$ & $<0.001$ \\
\hline \multicolumn{7}{|l|}{ EuroQol } \\
\hline EQ-5D index & $0.32(0.30,0.34)$ & $0.26(0.26,0.27)$ & $<0.001$ & $0.83(0.82,0.84)$ & $0.72(0.72,0.73)$ & $<0.001$ \\
\hline EQ VAS & $49(48,51)$ & $45(45,46)$ & $<0.001$ & $81(80,82)$ & $72(72,73)$ & $<0.001$ \\
\hline \multicolumn{7}{|l|}{ ODI } \\
\hline Index & $42(41,43)$ & $48(47,48)$ & $<0.001$ & $10(9,10)$ & $18(18,19)$ & $<0.001$ \\
\hline
\end{tabular}

Data are presented mean $(95 \% \mathrm{CI})$. NA = not applicable. NBP (No back pain) refers to patients without any preoperative back pain according to NRS $(n=1.333)$ and LBP (Low back pain) refers to patients with preoperative back pain NRS $>0(n=14,085)$

\section{Discussion}

The main indication for surgery for lumbar disc herniation is leg pain or sciatica. Spine surgeons have traditionally considered patients where the chief complaint is sciatica to be good candidates for surgery. This study confirms this longheld belief.

Our study shows leg pain without back pain to be a relatively common finding in patients scheduled for LDH surgery as almost one in ten reports no back pain whatsoever. The findings of our study suggest that the surgical outcome is more favorable in LDH patients if back pain is absent preoperatively. A substantially larger proportion of the NBP patients are pain free or much better with regards to leg pain after surgery compared with the LPB patients (87 vs $76 \%$ ).

Type of surgery may affect outcome at the one-year follow-up. In Swespine, surgery for LDH can be classified as discectomy with or without the use of microscope. 
Table 3 Preoperative demographics in patients with no preoperative back pain divided into patients with no significant postoperative significant back pain (NSPBP) and patients with significant postoperative back pain (defined as $>2.4$ NRS back pain) (SPBP)

\begin{tabular}{llll}
\hline Group & NPSBP & SPBP & Comparison \\
\hline Age & 42 & 42 & $p=0.94$ \\
Gender (M/F) & $61 / 39$ & $46 / 54$ & $\begin{array}{l}\boldsymbol{p}<\mathbf{0 . 0 0 1} \\
\boldsymbol{p}<\mathbf{0 . 0 5}\end{array}$ \\
Smoking & 12 & 20 & \\
NRS back & & & $\boldsymbol{p}<\mathbf{0 . 0 0 1}$ \\
$\quad$ Postop & $0.4(0.4,0.4)$ & $4.8(4.1,5.0)$ & \\
VAS leg & & & $\boldsymbol{p}<\mathbf{0 . 0 1}$ \\
Preop & $6.0(5.9,6.2)$ & $6.6(6.2,7.1)$ & $\boldsymbol{p}<\mathbf{0 . 0 0 1}$ \\
Postop & $1.0(0.9,1.1)$ & $3.0(2.5,3.4)$ & \\
EQ-index & & & $\boldsymbol{p}<\mathbf{0 . 0 1}$ \\
Preop & $0.33(0.30,0.35)$ & $0.25(0.20,0.30)$ & $\boldsymbol{p}<\mathbf{0 . 0 0 1}$ \\
Postop & $0.86(0.85,0.87)$ & $0.62(0.57,0.66)$ & \\
ODI & & & $\boldsymbol{p}<\mathbf{0 . 0 5}$ \\
Preop & $42(40,43)$ & $45(42,49)$ & $\boldsymbol{p}<\mathbf{0 . 0 0 1}$ \\
Postop & $7(6,8)$ & $27(24,29)$ & \\
\hline
\end{tabular}

Data are presented mean $(95 \% \mathrm{CI})$ or proportions (\%). Statistically significant differences are highlighted in bold

We have no information about if tubular or other minimally invasive approaches were conducted, i.e., endoscopic surgery. The same applies for information about the amount of disc material removed, which is not registered in Swespine. Aggressive discectomy may result in more back pain, but in current practice, most surgeons perform sequestrectomy.
However, also patients with concomitant preoperative back pain improve significantly following surgery in all PROMs in the present study. Patients in the NBP and LBP group achieve similar improvements, but the LBP group is left with more pronounced postoperative disability. These findings fit well into our current frame of reference as patients with LDH and high burden of back pain may be a more heterogeneous group of patients both in terms of symptoms and morphological disease. Although there are statistically significant differences between the NBP and the LBP groups at the one-year follow-up milestone, it should be noted that the differences are relatively small and may not consistently be clinically relevant.

To our knowledge, the cohort of LDH patients that report no preoperative back pain has not previously been selectively studied. The outcome following surgery in this cohort is reported with less preoperative disability reflected in better self-estimated walking distance, lower consumption of analgesics, better quality of life and less disability as compared to patients with preoperative LBP. The NBP group report increased back pain postoperatively as compared to preoperatively (13\% NBP vs 39\% LBP with back pain difference $>$ MCID). One possible explanation is that the disc herniation may represent the beginning of a symptomatic segmental disease of the spine. The degree of back pain present at one-year follow-up in patients without preoperative back pain is however probably of lesser importance since this is lower than the MCID for back pain in $87 \%$ of the patients after LDH surgery [14] and does not reflect in poor postoperative quality of life (SF-36, EQ-5D) or disability (ODI).
Table 4 Improvements by surgery from preoperative to one-year postoperative status in NRS as estimation of back and leg pain, EQ-5D and SF-36 as estimation of quality of life and ODI as estimation of disability

\begin{tabular}{|c|c|c|c|c|}
\hline & \multicolumn{4}{|c|}{ Improvement at oneyear postoperatively } \\
\hline & NBP & LBP & Comparison 1 & $\begin{array}{l}\text { NBP comparison }{ }^{2} \\
\text { LBP comparison }{ }^{2}\end{array}$ \\
\hline \multicolumn{5}{|l|}{ NRS } \\
\hline Leg pain & $5.0(4.8,5.2)$ & $4.7(4.7,4.8)$ & 0.005 & $\begin{array}{l}<0.001 \\
<0.001\end{array}$ \\
\hline Back pain & $-1(-0.9,-1.1)$ & $2.6(2.5,2.7)$ & $<0.001$ & $\begin{array}{l}<0.001 \\
<0.001\end{array}$ \\
\hline \multicolumn{5}{|l|}{ EuroQol } \\
\hline EQ-5D index & $0.51(0.49,0.53)$ & $0.46(0.45,0.46)$ & $<0.001$ & $\begin{array}{l}<0.001 \\
<0.001\end{array}$ \\
\hline EQ VAS & $32(30,33)$ & $27(27,28)$ & $<0.001$ & $\begin{array}{l}<0.001 \\
<0.001\end{array}$ \\
\hline \multicolumn{5}{|l|}{ ODI } \\
\hline Index & $32(31,33)$ & $29(29,30)$ & $<0.001$ & $\begin{array}{l}<0.001 \\
<0.001\end{array}$ \\
\hline
\end{tabular}

Data are presented as mean with $95 \%$ confidence interval within brackets. NBP (No back pain) refers to patients without any preoperative back pain according to NRS $(n=1333)$ and LBP (Low back pain) refers to patients with preoperative back pain NRS $>0(n=14,085)$. Comparison ${ }^{1}$ refers to comparison in improvement following surgery between group NBP and LBP; comparison ${ }^{2}$ refers to comparison from before to after surgery within group NBP and LBP 
We found some baseline differences in patients that were preoperatively without back pain but subsequently developed significant back pain at the one-year follow-up and those who remained pain free. Female sex and smoking were associated with increased risk for having clinically significant back pain at the one-year follow-up. Furthermore, patients that developed clinically significant back pain at the one-year follow-up had preoperatively more disability in terms of the ODI and lower quality of life in terms of the EQ-5D. However, the baseline differences in ODI and EQ-5D in these patient groups were small and not clinically significant.

Patients with LDH without back pain are good candidates for surgery. The increased knowledge regarding this patient population can be used by surgeons preoperatively when discussing surgery with patients with LDH. It allows for more nuanced preoperative information and patients may thus attain well founded expectations with regards to the outcome of surgery. Outcome of surgery in well-informed patients is associated with a better surgical result and a higher degree of patient satisfaction $[11,12]$.

Shortcomings of the present study those associated with register studies such as the obvious inclusion bias and the retrospective analysis of prospectively collected data. However, the study includes a very large number of patients in the setting of a nationwide span with a heterogenous study population as regarding patient selection and comorbidities. In the Swespine, only the reason for surgery is registered, but the individual patients may have other spinal conditions and or psychosomatic conditions that may impact the outcome of surgery. These potential comorbidities remain unaccounted for. Another shortcoming of the present study is the absence of information regarding preoperative non-surgical treatment which may influence the outcome of surgery. Regular practice in Sweden is to offer non-operative treatment (analgesics and physiotherapy) of symptomatic LDH for at least 8-week period before surgery is considered.

The transition question for evaluation of leg pain outcome may be affected by recall bias and there are previous reports that show that perceived change is influenced by the current health state. Strengths of this study include a large patient material and the use of validated PROMs. 29\% of the patients did not have complete data and were excluded; however, a loss of $30 \%$ have been reported to not influence the outcome in spine register studies [15], but conflicting opinions has also been reported [16].

To conclude, patients with no preoperative back pain seem to have a better outcome following LDH as compared to patients with concomitant back pain and with a higher degree of patient satisfaction. $13 \%$ of the patients where back pain is absent preoperatively develop clinically significant back pain at the one-year follow-up.
Funding Open access funding provided by Örebro University.

Open Access This article is licensed under a Creative Commons Attribution 4.0 International License, which permits use, sharing, adaptation, distribution and reproduction in any medium or format, as long as you give appropriate credit to the original author(s) and the source, provide a link to the Creative Commons licence, and indicate if changes were made. The images or other third party material in this article are included in the article's Creative Commons licence, unless indicated otherwise in a credit line to the material. If material is not included in the article's Creative Commons licence and your intended use is not permitted by statutory regulation or exceeds the permitted use, you will need to obtain permission directly from the copyright holder. To view a copy of this licence, visit http://creativecommons.org/licenses/by/4.0/.

\section{References}

1. Stromqvist B, Fritzell P, Hagg O, Jonsson B, Sanden B, Swedish Society of Spinal S (2013) Swespine: the Swedish spine register: the 2012 report. Eur Spine J 22:953-974. doi: https://doi.org/10.1007/ s00586-013-2758-9

2. Peul WC, van Houwelingen HC, van den Hout WB, Brand R, Eekhof JA, Tans JT, Thomeer RT, Koes BW, Leiden-The Hague Spine Intervention Prognostic Study G (2007) Surgery versus prolonged conservative treatment for sciatica. N Engl J Med 356:2245-2256. doi: https://doi.org/10.1056/NEJMoa064039

3. Spangfort EV (1972) The lumbar disc herniation. A computer-aided analysis of 2504 operations. Acta Orthop Scand Suppl 142:1-95

4. Weinstein JN, Tosteson TD, Lurie JD, Tosteson AN, Hanscom B, Skinner JS, Abdu WA, Hilibrand AS, Boden SD, Deyo RA (2006) Surgical vs nonoperative treatment for lumbar disk herniation: the Spine Patient Outcomes Research Trial (SPORT): a randomized trial. JAMA 296:2441-2450. https://doi.org/10.1001/jama.296.20. 2441

5. Stromqvist F, Ahmad M, Hildingsson C, Jonsson B, Stromqvist B (2008) Gender differences in lumbar disc herniation surgery. Acta Orthopaed 79:643-649. https://doi.org/10.1080/174536708100166 69

6. Stromqvist F, Stromqvist B, Jonsson B, Gerdhem P, Karlsson MK (2015) Outcome of surgical treatment of lumbar disc herniation in young individuals. Bone Joint J 97-B:1675-1682. doi: https://doi. org/10.1302/0301-620X.97B12.36258

7. Stromqvist F, Stromqvist B, Jonsson B, Karlsson MK (2016) Inferior outcome of lumbar disc surgery in women due to inferior preoperative status: a prospective study in 11,237 patients. Spine 41:12471252. https://doi.org/10.1097/BRS.0000000000001492

8. Hareni N, Stromqvist F, Stromqvist B, Rosengren BE, Karlsson MK (2019) Predictors of satisfaction after lumbar disc herniation surgery in elderly. BMC Musculoskelet Disord 20:594. https://doi.org/10. 1186/s12891-019-2975-4

9. Hareni N, Stromqvist F, Stromqvist B, Sigmundsson FG, Rosengren BE, Karlsson MK (2021) Back pain is also improved by lumbar disc herniation surgery. Acta Orthopaed 92:4-8. https://doi.org/10.1080/ 17453674.2020.1815981

10. Stromqvist F, Stromqvist B, Jonsson B, Karlsson MK (2016) Gender differences in patients scheduled for lumbar disc herniation surgery: a National Register Study including 15,631 operations. Eur Spine J 25:162-167. https://doi.org/10.1007/s00586-015-4052-5

11. Krupic F, Rolfson O, Nemes S, Karrholm J (2016) Poor patientreported outcome after hip replacement, related to poor perception of perioperative information, commoner in immigrants than in nonimmigrants. Acta Orthopaed 87:218-224. https://doi.org/10.3109/ 17453674.2015.1135664

12. Ronnberg K, Lind B, Zoega B, Halldin K, Gellerstedt M, Brisby H (2007) Patients' satisfaction with provided care/information and 
expectations on clinical outcome after lumbar disc herniation surgery. Spine 32:256-261. https://doi.org/10.1097/01.brs.0000251876. 98496.52

13. Parai C, Hagg O, Lind B, Brisby H (2018) The value of patient global assessment in lumbar spine surgery: an evaluation based on more than 90,000 patients. Eur Spine J 27:554-563. https://doi.org/ 10.1007/s00586-017-5331-0

14. Solberg T, Johnsen LG, Nygaard OP, Grotle M (2013) Can we define success criteria for lumbar disc surgery? Estimates for a substantial amount of improvement in core outcome measures. Acta Orthopaed 84:196-201. https://doi.org/10.3109/17453674.2013.786634

15. Solberg TK, Sorlie A, Sjaavik K, Nygaard OP, Ingebrigtsen T (2011) Would loss to follow-up bias the outcome evaluation of patients operated for degenerative disorders of the lumbar spine? Acta Orthopaed 82:56-63. https://doi.org/10.3109/17453674.2010.548024

16. Parai C, Hagg O, Willers C, Lind B, Brisby H (2020) Characteristics and predicted outcome of patients lost to follow-up after degenerative lumbar spine surgery. Eur Spine J 29:3063-3073. https://doi. org/10.1007/s00586-020-06528-y

Publisher's Note Springer Nature remains neutral with regard to jurisdictional claims in published maps and institutional affiliations. 